\title{
Energy backtransfer and infrared photoresponse in erbium-doped silicon $\boldsymbol{p}-\boldsymbol{n}$ diodes
}

\author{
N. Hamelin, ${ }^{\text {a) }}$ P. G. Kik, J. F. Suyver, ${ }^{\text {b) }}$ K. Kikoin, ${ }^{\text {() }}$ and A. Polman ${ }^{\text {d) }}$ \\ FOM Institute for Atomic and Molecular Physics, Kruislaan 407, 1098 SJ Amsterdam, The Netherlands
}

\author{
A. Schönecker and F. W. Saris \\ Netherlands Energy Research Foundation ECN, P.O. Box 1, 1755 ZG Petten, The Netherlands
}

(Received 23 June 1999; accepted for publication 20 July 2000)

\begin{abstract}
Temperature-dependent measurements of the photoluminescence (PL) intensity, PL lifetime, and infrared photocurrent, were performed on an erbium-implanted silicon $p-n$ junction in order to investigate the energy transfer processes between the silicon electronic system and the Er $4 f$ energy levels. The device features excellent light trapping properties due to a textured front surface and a highly reflective rear surface. The PL intensity and PL lifetime measurements show weak temperature quenching of the erbium intra- $4 f$ transition at $1.535 \mu \mathrm{m}$ for temperatures up to $150 \mathrm{~K}$, attributed to Auger energy transfer to free carriers. For higher temperatures, much stronger quenching is observed, which is attributed to an energy backtransfer process, in which Er deexcites by generation of a bound exciton at an Er-related trap. Dissociation of this exciton leads to the generation of electron-hole pairs that can be collected as a photocurrent. In addition, nonradiative recombination takes place at the trap. It is shown for the first time that all temperature-dependent data for PL intensity, PL lifetime, and photocurrent can be described using a single model. By fitting all temperature-dependent data simultaneously, we are able to extract the numerical values of the parameters that determine the (temperature-dependent) energy transfer rates in erbium-doped silicon. While the external quantum efficiency of the photocurrent generation process is small $\left(1.8 \times 10^{-6}\right)$ due to the small erbium absorption cross section and the low erbium concentration, the conversion of Er excitations into free $e-h$ pairs occurs with an efficiency of $70 \%$ at room temperature. () 2000 American Institute of Physics. [S0021-8979(00)09620-1]
\end{abstract}

\section{INTRODUCTION}

Erbium-doped silicon is an interesting material, in which energy transfer can take place between the semiconductor electronic system and the internal $4 f$ states of the rare earth ion. ${ }^{1-7}$ Previously, this material has mainly been studied because it provides a means to attain light emission from silicon, a phenomenon that is of great importance in Si-based optoelectronic technology. Indeed, room-temperature photoluminescence (PL) from Er-doped silicon has been reported, and room-temperature light-emitting diodes have been fabricated. ${ }^{8}$ The excitation mechanism of $\mathrm{Er}$ in $\mathrm{Si}$ can be described in terms of an impurity Auger excitation process as indicated schematically in Fig. 1. Electron-hole $(e-h)$ pairs, generated either optically or electrically, can be trapped at an Er-related defect level ( $W_{T}$ in Fig. 1). Subsequently, the $e-h$ pair can recombine and energy is transferred to the Er ion ( $W_{E}$ in Fig. 1), which then becomes excited from the ground state $\left({ }^{4} I_{15 / 2}\right)$ to the first excited state $\left({ }^{4} I_{13 / 2}\right)$. The Er may then decay ( $W_{0}$ in Fig. 1$)$ by the emission of a photon with a wavelength of $1.54 \mu \mathrm{m}$.

It is well known that the photo- and electroluminescence from Er in Si both decrease strongly ("quench") as the tem-

\footnotetext{
a) Present address: ST Microelectronics, Catania, Italy.

${ }^{b}$ Present address: Debye Institute, Utrecht University, The Netherlands.

${ }^{c}$ Permanent address: Weizmann Institute of Science, Rehovot, Israel.

d)Electronic mail: polman@amolf.nl
}

perature is increased. ${ }^{2,5,6,8}$ Two quenching mechanisms have been identified. First, at temperatures typically above $30 \mathrm{~K}$, Auger quenching takes place, ${ }^{4}$ in which an excited Er ion is deexcited by energy transfer to a free electron or hole $\left(W_{A, e}\right.$ and $W_{A, h}$ in Fig. 1). The strength of this process depends on the free carrier concentration, which in turn depends on the dopant concentration and the temperature. Second, at temperatures typically above $150 \mathrm{~K}$ a much stronger quenching process becomes significant, which is attributed to an energy backtransfer process characterized by a rate constant $W_{\mathrm{BT}}$ as shown in Fig. 1. In this process an excited Er ion decays by generation of an $e-h$ pair bound at an Er-related trap. This backtransfer process is the reverse of the excitation process $W_{E}$ depicted in Fig. 1. Once the bound $e-h$ pair is formed, it can recombine and retransfer its energy to the Er ion or it can dissociate, either by absorption of a photon or by absorbing energy from lattice vibrations, leading to the generation of a free electron-hole pair (rate constant $W_{D}$ in Fig. 1).

At present this energy backtransfer mechanism from an excited $\mathrm{Er}$ ion to the Si electronic system limits the roomtemperature performance of erbium-doped silicon photo- and electroluminescence devices. On the other hand, the backtransfer process may also be used in an advantageous way, namely to detect infrared light in silicon. Normally, silicon only weakly absorbs infrared light at photon energies below 


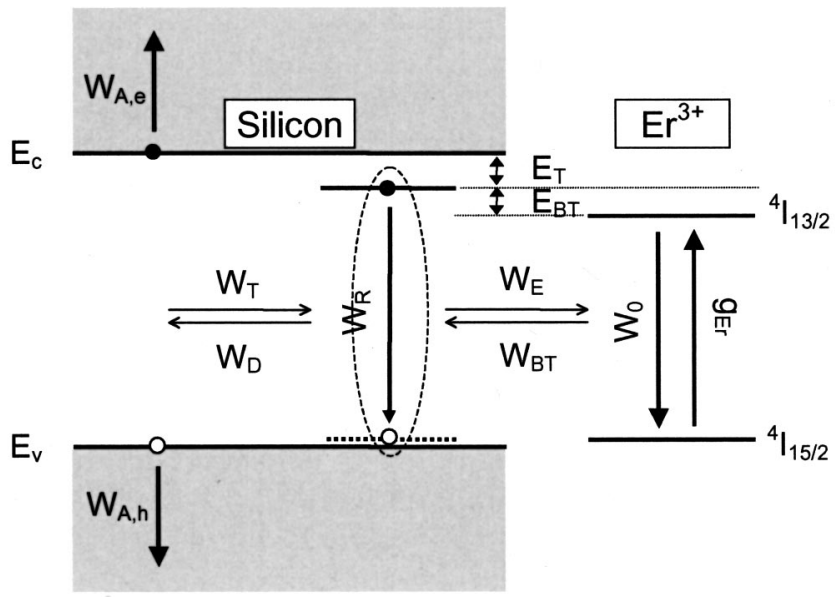

FIG. 1. Energy transfer processes in Er-doped silicon. Optically generated carriers in Si can be trapped at an Er-related trap level in the Si band gap. The bound exciton may then recombine and transfer its energy to the $\mathrm{Er}^{3+}$ ion, which can then emit a photon at $1.535 \mu \mathrm{m}$. In the reverse process, an optically excited Er ion decays by the creation of a bound exciton, which can then dissociate resulting in the generation of a free electron-hole pair. In a $p-n$ junction geometry these carriers can be collected leading to a net photocurrent at $1.534 \mu \mathrm{m}$. Exciton recombination at the Er-related trap is also included.

the band gap at $1.1 \mathrm{eV} .{ }^{9}$ However, if the silicon is doped with Er, $1.54 \mu \mathrm{m}$ photons may be absorbed by the Er, which in turn can generate free carriers via the backtransfer process as described previously, followed by the dissociation process. If the Er is located in or near the depletion region of a $p-n$ junction these free carriers generate a current, an effect known as the impurity photovoltaic effect. Early demonstrations of an Er-related photocurrent can be found in Refs. 6, 10 , and 11, in which $\mathrm{Si} p-n$ junctions show an enhanced photoresponse around $1.53 \mu \mathrm{m}$ as a result of Er doping. Based on this process, Er doping of silicon may be used to improve the efficiency of Si-based solar cells or to fabricate Si-based infrared detectors.

Although all processes in Fig. 1 are known, little is known about their relative importance. In particular, the parameters $W_{E}, W_{R}$, as well as the absorption cross section of $\mathrm{Er}$ in $\mathrm{Si}\left(\sigma_{1.535}\right)$ which are all needed to predict the quantum efficiency of an Er-doped $p-n$ junction, are not known. In this article, we perform a quantitative study of all excitation and deexcitation processes in Er-doped Si. Temperaturedependent measurements of the $1.54 \mu \mathrm{m}$ PL intensity, PL lifetime, and photocurrent are performed on an erbiumimplanted Si $p-n$ junction. By comparing the data with calculations using a rate-equation model that takes into account all energy transfer processes as depicted in the model of Fig. 1 the energy transfer rates and activation energies are derived. It is found that after optical excitation of the Er, the generation of free carriers that can be collected (i.e., backtransfer followed by exciton dissociation) occurs with an efficiency of $70 \%$ at room temperature. At $300 \mathrm{~K}$, the ratelimiting step in this process is the backtransfer. All these measurements were only possible because of the use of a specially textured solar cell geometry, in which enhanced light trapping significantly enhanced the effective optical excitation rate of $\mathrm{Er}$ ions in the $p-n$ junction. Finally, the implications of this work for solar cell and infrared detector technology are addressed.

\section{EXPERIMENT}

A 200- $\mu$ m-thick float zone grown silicon (100) wafer (B doped, resistivity $2.5 \Omega \mathrm{cm}$ ) was processed for the fabrication of a passivated-emitter rear-locally-diffused (PERL) solar cell ${ }^{10}$ at the University of New South Wales. To enhance the light trapping in the cell, the wafer top surface was textured by anisotropic etching, yielding square-based inverted pyramids with facets inclined at $55^{\circ}$ from the sample normal. Next, 3.5 MeV Er ions were implanted into the textured surface layer to a total dose of $1 \times 10^{13}$ ions $/ \mathrm{cm}^{2}$. The emitter region was formed by surface doping with phosphorous, carried out from a gas source in a tube furnace, followed by thermal annealing at $835^{\circ} \mathrm{C}$ and two oxidation steps at $1030^{\circ} \mathrm{C}$ for $60 \mathrm{~min}$ and $1000^{\circ} \mathrm{C}$ for $75 \mathrm{~min}$. During these high temperature processes most of the damage generated during implantation was annealed out. The surface $\mathrm{P}$ concentration after annealing was $1 \times 10^{19}$ ions $/ \mathrm{cm}^{3}$, with the emitter region extending to a depth of $0.7 \mu \mathrm{m}$, measured normal to the facets. The Er projected range and straggle are 1.0 and $0.2 \mu \mathrm{m}$, respectively. The mean implant range normal to the facets then is $0.6 \mu \mathrm{m}$, placing Er near the junction region of the cell. Part of Er will therefore be located in the space charge region of the junction, while the remaining Er will be located in the emitter and base regions of the cell.

Current-voltage measurements on the Er-implanted PERL cell show an open circuit voltage of $0.664 \mathrm{~V}$, a fill factor of 0.74 , and an efficiency of $19.2 \%$ under AM1.5 illumination. This is lower than the typical efficiency for standard PERL cells, which is attributed to carrier recombination at defect levels caused by remaining ion implantation damage. The cell was placed in a closed-cycle helium cryostat using silver paint to ensure good electrical and thermal contact. Infrared photocurrent measurements were done in short circuit condition using a tunable solid state laser diode with a wavelength ranging from 1.50 to $1.58 \mu \mathrm{m}$ at a power of typically $1 \mathrm{~mW}$, and spectral resolution of $0.01 \mathrm{~nm}$. The area of the laser spot on the sample was $1.5 \mathrm{~cm}^{2}$. In the same setup, the $1.54 \mu \mathrm{m}$ photoluminescence resulting from excitation with the $515 \mathrm{~nm}$ line of an Ar ion laser was measured. A $48 \mathrm{~cm}$ monochromator was used in combination with a liquid-nitrogen-cooled Ge detector and phase sensitive detection techniques. The spectral resolution was $6 \mathrm{~nm}$. The 1/e PL lifetime was measured by modulating the pump beam on-off and recording the time-dependent luminescence signal on a digital oscilloscope.

\section{RESULTS}

Figure 2 shows an infrared photocurrent spectrum measured on the textured PERL solar cell at $300 \mathrm{~K}$ under 1.5 $\mathrm{mW}$ irradiation. The spectrum displays the characteristic features of an Er absorption spectrum peaking around 1.534 $\mu \mathrm{m}$. The short circuit current at $300 \mathrm{~K}$ was $3.3 \mathrm{nA}$ at 1.534 $\mu \mathrm{m}$. The external quantum efficiency, defined as the number of collected $e-h$ pairs per incoming photon, thus is 1.8 $\times 10^{-6}$ at $1.534 \mu \mathrm{m}$. Note that due to the textured top sur- 


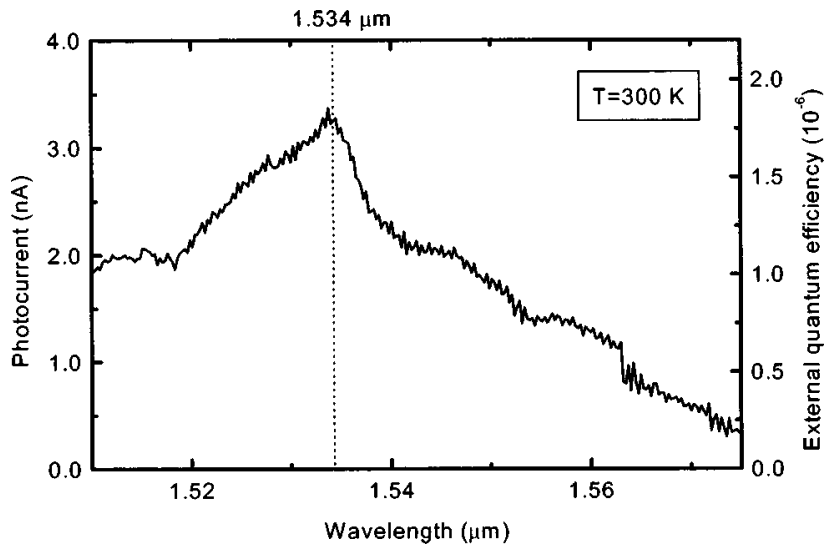

FIG. 2. Photocurrent spectrum of a PERL Si solar cell implanted with Er at $3.5 \mathrm{MeV}$ to a dose of $1 \times 10^{13} \mathrm{ions} / \mathrm{cm}^{2}$. An external quantum efficiency scale is provided on the right-hand side.

face and the highly reflective backside of the PERL cell, each incoming photon experiences multiple internal reflections and will therefore pass several times through the Erdoped region. In fact, similar experiments on untextured $\mathrm{Si}$ solar cells showed a photocurrent at $1.534 \mu \mathrm{m}$ at $300 \mathrm{~K}$ of only $0.5 \mathrm{nA}$, seven times lower than the PERL cell. At this low current the signal-to-noise ratio was too poor to measure the temperature dependence of the photocurrent of this planar cell such as will be shown in the following for the PERL cell.

Figure 3 shows the temperature dependence of the photocurrent at $1.534 \mu \mathrm{m}$, plotted in an Arrhenius graph. It can clearly be seen that the photocurrent increases over nearly two orders of magnitude, as the temperature is increased from 100 to $300 \mathrm{~K}$. Also plotted in Fig. 3 is the photoluminescence at $1.535 \mu \mathrm{m}$ measured on the same sample, under $515 \mathrm{~nm}$ excitation. Three distinct temperature regions can be identified for the PL intensity as a function of temperature: (I) a temperature-independent region below $30 \mathrm{~K}$, (II) a weakly temperature-dependent region between 30 and 150 $\mathrm{K}$, and (III) a strongly temperature-dependent decrease of the

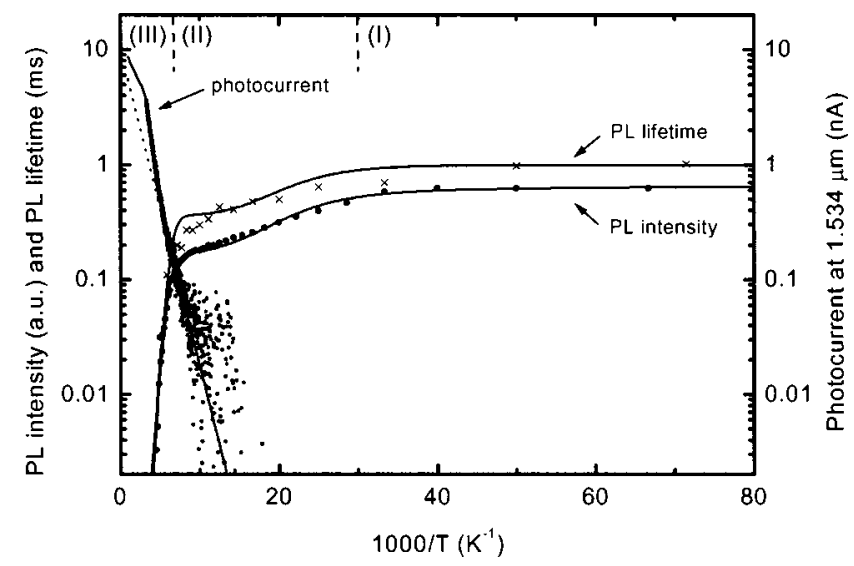

FIG. 3. Temperature-dependent photocurrent at $1.534 \mu \mathrm{m}$, measured on the Er-implanted PERL cell. The temperature dependence of photoluminescence intensity and PL lifetime at $1.535 \mu \mathrm{m}$ excited by $515 \mathrm{~nm}$ light are also included. Three quenching regimes are indicated, and are labeled (I), (II), and (III). The drawn lines are fits of the model, using the parameters in Table I.

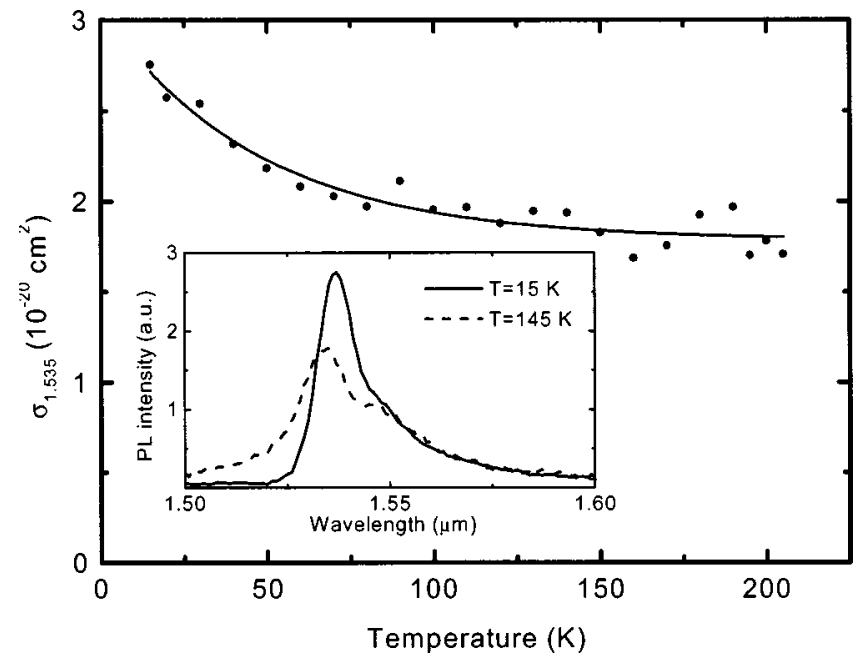

FIG. 4. Temperature dependence of the Er emission cross section at 1.535 $\mu \mathrm{m}$, derived from PL spectra such as shown in the inset (taken at 15 and 145 $\mathrm{K})$. The line drawn through the data is described by Eq. (1).

intensity above $150 \mathrm{~K}$. Figure 3 also shows the temperature dependence of the PL lifetime at $1.535 \mu \mathrm{m}$, which shows the same trend as the PL intensity. Note that a measurable photocurrent is only observed in the temperature range where both PL intensity and PL lifetime show strong temperature quenching.

Spectral photocurrent measurements at temperatures below $150 \mathrm{~K}$ show a small signal, not related to Er, most likely due to implantation-induced defects in the $p-n$ junction. Indeed it has been shown that implantation damage induces an infrared photoresponse in silicon solar cells. ${ }^{10}$ The observed defect current can be described by an activated process, with an activation energy of $55 \mathrm{meV}$, as indicated by the dotted line in Fig. 3.

\section{ENERGY TRANSFER PROCESSES}

In order to calculate and predict the temperature dependence of the PL intensity and PL lifetime as well as the photocurrent, the following five processes must be taken into account.

\section{A. Optical absorption and emission $\left(\sigma_{1.535}\right)$}

$\mathrm{The} \mathrm{Er}^{3+}$ emission cross section is temperature dependent, due to changes in the thermal distribution over the Stark levels in the excited state manifold of $\mathrm{Er}^{3+}$ as the temperature is increased. This can be seen by comparing the normalized PL emission spectra taken at 15 and $145 \mathrm{~K}$, shown in the inset in Fig. 4. This effect must be included in the analysis of both the infrared photocurrent and of the photoluminescence data.

From the shape of the PL spectra, the optical emission cross section of Er in Si can be calculated, ${ }^{12}$ assuming that the measured PL lifetime at $15 \mathrm{~K}(1.0 \mathrm{~ms}$, see Fig. 3$)$ is equal to the radiative lifetime of Er in Si. From such a calculation, using the PL spectrum taken at $15 \mathrm{~K}$ it follows that the emission cross section at $15 \mathrm{~K}$ at $1.535 \mu \mathrm{m}$ equals 2.7 $\times 10^{-20} \mathrm{~cm}^{2}$. 
The temperature dependence of the Er emission cross section at $1.535 \mu \mathrm{m}$ can be determined by taking normalized measurements of the integrated PL emission spectrum as a function of temperature, as shown in Fig. 4. The drawn line is a smooth fit through the data described by

$$
\sigma_{1.535}(T)\left(10^{-20} \mathrm{~cm}^{2}\right)=1.8+1.2 \exp (-0.02 \times T(\mathrm{~K})) .
$$

From the McCumber reciprocity formula ${ }^{13}$ it follows that the temperature dependence of the absorption cross section is similar to that of the emission cross section at the location of the emission peak. Moreover, it is generally observed that the magnitude of the absorption cross section at this wavelength is close to the emission cross section. The rate constant for optical excitation of Er under $1.534 \mu \mathrm{m}$ illumination is then given by

$$
g_{\mathrm{Er}}=(1-R) L_{\mathrm{eff}} \sigma_{1.535} \phi,
$$

with $\phi$ the photon flux $\left(\mathrm{cm}^{-2} \mathrm{~s}^{-1}\right), R$ the reflectivity of the cell around $1.535 \mu \mathrm{m}$ (taken equal to zero), and $L_{\text {eff }}$ the effective number of light passes through the depletion region. This rate constant is defined as the probability per unit time that an Er ion in the ground state is optically excited.

\section{B. Auger quenching $\left(W_{A, e}, W_{A, h}\right)$}

An excited Er ion can decay by Auger energy transfer to free carriers. Such carriers are provided by the $\mathrm{P}$ doping in the emitter region of the cell, the B doping of the Si substrate, and by the Er ions which can act as a donor in $\mathrm{Si}^{14}$ The Auger quenching effect causes a decrease in the PL lifetime and in the luminescence intensity, but does not generate free $e-h$ pairs. The Auger quenching rate constant to free electrons in erbium-doped silicon containing a donor concentration $N_{D}\left(\mathrm{~cm}^{-3}\right)$ is given by

$$
W_{A, e}(T)=C_{A, e} N_{D}\left(1+2 \exp \left(-\frac{E_{d}-E_{F}}{k T}\right)\right)^{-1},
$$

where $C_{A, e}\left(\mathrm{~cm}^{-3} \mathrm{~s}^{-1}\right)$ is the Auger quenching coefficient for free electrons, $E_{d}$ the donor ionization energy, and $E_{F}$ the Fermi energy. For Auger quenching to free holes, a similar relation holds. The Auger quenching coefficient for $\mathrm{Er}$ in $\mathrm{Si}$ has recently been measured by Priolo et al. ${ }^{5}$ and amounts to $5 \times 10^{-13} \mathrm{~cm}^{3} \mathrm{~s}^{-1}$ for both electrons and holes. In the fitting procedure we vary $N_{D}$ and $E_{d}$, while the Fermi energy as a function of temperature is numerically determined.

\section{Er excitation and backtransfer $\left(W_{E}, W_{\mathrm{BT}}\right)$}

Erbium can be excited by recombination of a trapped $e-h$ pair at an Er-related defect level in the Si band gap (see Fig. 1). The temperature-independent rate constant for this process is denoted as $W_{E}$. Conversely, an excited Er ion can decay nonradiatively by the formation of such a trapped $e-h$ pair. The temperature dependence of the backtransfer process may be described either by a single activation barrier or by a phonon-assisted process. ${ }^{15}$ In both cases, the rate constant for energy backtransfer becomes

$$
W_{\mathrm{BT}}(T)=W_{E} \exp \left(-\frac{E_{\mathrm{BT}}}{k T}\right) .
$$

Previous measurements on the PL lifetime quenching of $\mathrm{Er}$ in silicon have indicated a backtransfer energy of $150 \mathrm{meV}$ at temperatures around $150 \mathrm{~K}^{6}$ As the band gap of silicon decreases by $45 \mathrm{meV}$ upon going from 15 to $300 \mathrm{~K},{ }^{16}$ the trap energy will most likely be temperature dependent. The depth of the trap as a function of temperature is assumed to show the same relative variation as the silicon band gap.

\section{Electron-hole pair trapping and dissociation $\left(W_{D}, W_{T}\right)$}

The rate constant for carrier trapping at an Er-related trap is given by $W_{T}=\sigma_{T} v_{\text {th }} N_{T}$, with $\sigma_{T}$ a temperatureindependent carrier trapping cross-section, $v_{\text {th }}$ the carrier thermal velocity given by $\left(3 k T / m_{e, h}\right)^{1 / 2}$, and $N_{T}\left(\mathrm{~cm}^{-3}\right)$ the trap density, which we assume to be equal to the Er density. Once an $e-h$ pair is trapped, it can recombine and excite the Er ion, or it can dissociate with an activation energy $E_{T}$, generating a free electron-hole pair. Inside the depletion region these carriers are immediately separated by the built-in electric field $\left(\sim 10^{4} \mathrm{~V} / \mathrm{cm}\right)$. Assuming that photon-assisted dissociation is negligible at the low applied photon flux (5 $\times 10^{15} \mathrm{~cm}^{-2} \mathrm{~s}^{-1}$ ), the rate constant for the $e-h$ pair dissociation process is given by

$$
W_{D}(T)=\sigma_{T} v_{\mathrm{th}} N_{c} \exp \left(-\frac{E_{T}}{k T}\right) .
$$

The dissociation energy $E_{T}$ is given by the temperaturedependent difference between the trap energy and the conduction band. The description of backtransfer and detrapping imposes that the total energy of the thermal processes leading to free carrier generation corresponds to the silicon band gap: $E_{\mathrm{Er}}+E_{\mathrm{BT}}+E_{T}=E_{g}$, with $E_{\mathrm{Er}}$ the energy of the Er intra$4 f$ transition $(809 \mathrm{meV})$ and $E_{g}$ the temperature-dependent band gap of Si.

\section{E. Carrier recombination at the Er-related trap level}

The Er PL intensity is found to quench strongly at temperatures above $150 \mathrm{~K}$. The PL lifetime seems to follow this behavior, although the PL intensity quickly becomes too low to determine the PL lifetime. This type of PL lifetime quenching is generally observed for Er-doped silicon, 2,5,6 showing that at these temperatures a large fraction of the Er decay is occurring nonradiatively. If these nonradiative events are generating current, the infrared photocurrent should saturate already well below $300 \mathrm{~K}$. The fact that no such saturation is observed (see Fig. 3) implies that the initial PL lifetime quenching at temperatures around $150 \mathrm{~K}$ is due to nonradiative decay processes that do not generate free $e-h$ pairs. To account for this effect, an additional recombination process must be included in the model of Fig. 1, of which the associated rate constant is denoted as $W_{R}$. This is an important observation, since this recombination process has not been included in any of the previous models ${ }^{3,5}$ to describe the temperature dependence of the Er-related PL in 
TABLE I. Input parameters for the model used to calculate the PL intensity, PL lifetime, and photocurrent data in Fig. 3.

\begin{tabular}{llll}
\hline \hline Parameter & Symbol & \multicolumn{1}{c}{ Value } & \multicolumn{1}{c}{ Source } \\
\hline Er areal density & $N_{\mathrm{Er}}$ & $1 \times 10^{13} \mathrm{~cm}^{-2}$ & This work \\
Photon Flux & $\phi$ & $7.7 \times 10^{15} \mathrm{~cm}^{-2} \mathrm{~s}^{-1}$ & This work \\
Si indirect band gap (15-300 K) & $E_{g}$ & $1.168-1.123 \mathrm{eV}$ & Ref. 16. \\
Er absorption/emission cross section (15 K) & $\sigma_{1.535}$ & $2.7 \times 10^{-20} \mathrm{~cm}^{2}$ & This work, Fig. 4 \\
Er radiative decay rate & $W_{0}$ & $1.0 \times 10^{3} \mathrm{~s}^{-1}$ & This work, Fig. 3 \\
Backtransfer prefactor & $W_{E}$ & $2 \times 10^{8} \mathrm{~s}^{-1}$ & This work (fitted) \\
Backtransfer activation energy (150 K) & $E_{\mathrm{BT}}$ & $150 \mathrm{meV}$ & Ref. 6 \\
Carrier trapping cross section & $\sigma_{T}$ & $3 \times 10^{-15} \mathrm{~cm}^{2}$ & Set \\
Dissociation activation energy (150 K) & $E_{T}$ & $199 \mathrm{meV}^{-}$ & Set at $E_{g}-\left(E_{\mathrm{BT}}+E_{\mathrm{Er}}\right)$ \\
Auger quenching constant & $C_{A}$ & $5 \times 10^{-13} \mathrm{~cm}^{3} \mathrm{~s}^{-1}$ & Ref. 5 \\
Auger quenching activation energy & $E_{A, h}$ & $30 \mathrm{meV}^{15}$ & This work (fitted) \\
Dopant concentration & $N_{D}$ & $3.5 \times 10^{15} \mathrm{~cm}^{-3}$ & This work (fitted) \\
Trap recombination rate & $W_{R}$ & $2 \times 10^{8} \mathrm{~s}^{-1}$ & This work (fitted) \\
Light trapping $\times$ collection efficiency & $L_{\text {eff }} \eta_{\text {coll }}$ & 9 & This work (fitted) \\
\hline \hline
\end{tabular}

$\mathrm{Si}$. As no PL is observed at the energy characteristic for this transition $(0.96 \mathrm{eV} ; 1.3 \mu \mathrm{m})$, it must be assumed that this recombination occurs nonradiatively.

\section{COMPARISON BETWEEN MODEL AND EXPERIMENT}

\section{A. Photoluminescence rate equations}

In PL measurements, the Er is excited through optically generated carriers (see Fig. 1). In the depletion region, these carriers are immediately swept away, which means that the PL signal mostly reflects Er located outside the depletion region. We will assume that a fixed fraction of the generated minority carriers is captured at the Er-related trap, resulting in a temperature-independent trapped $e-h$ pair generation rate, with a rate constant $g_{e h}$. The concentration of trapped electron-hole pairs $N_{e h}\left(\mathrm{~cm}^{-3}\right)$ and excited $\mathrm{Er}$ ions $N_{\mathrm{Er}}^{*}\left(\mathrm{~cm}^{-3}\right)$ resulting from this excitation are then determined by the following coupled rate equations:

$$
\begin{aligned}
& \frac{\partial N_{e h}}{\partial t}=g_{e h}+W_{\mathrm{BT}} N_{\mathrm{Er}}^{*}-\left(W_{E}+W_{D}+W_{R}\right) N_{e h}, \\
& \frac{\partial N_{\mathrm{Er}}^{*}}{\partial t}=W_{E} N_{e h}-\left(W_{0}+W_{A}+W_{\mathrm{BT}}\right) N_{\mathrm{Er}}^{*} .
\end{aligned}
$$

\section{B. Photoresponse rate equations}

In the case of photocurrent measurements, the Er ions are excited directly by $1.534 \mu \mathrm{m}$ light, at a rate $g_{\mathrm{Er}}$. Moreover, as the photocurrent is primarily generated by Er located within the depletion region, Auger quenching to free carriers is expected to be negligible. The rate equations then become

$$
\begin{aligned}
& \frac{\partial N_{e h}}{\partial t}=W_{\mathrm{BT}} N_{\mathrm{Er}}^{*}-\left(W_{E}+W_{D}+W_{R}\right) N_{e h}, \\
& \frac{\partial N_{\mathrm{Er}}^{*}}{\partial t}=g_{\mathrm{Er}} N_{\mathrm{Er}}+W_{E} N_{e h}-\left(W_{0}+W_{\mathrm{BT}}\right) N_{\mathrm{Er}}^{*} .
\end{aligned}
$$

Both sets of differential equations were solved analytically for steady state conditions.

\section{Model calculations}

All experimental data in Fig. 3 can be described as a function of temperature by solving Eqs. (6)-(9): The photocurrent is given by $q N_{e h}(T) \times W_{D}(T) \times V \times \eta_{\text {coll }}$ with $q$ the electron charge, $V$ the illuminated volume of the depletion region, and $\eta_{\text {coll }}$ the carrier collection efficiency, which for visible radiation was measured to be $\sim 1$ for all temperatures probed, the PL intensity is proportional to $\sigma_{1.535}(T)$ $\times N_{\mathrm{Er}}^{*}(T)$, and the photoluminescence lifetime is to first order given by

$$
\tau_{\mathrm{PL}}(T)=\left[W_{0}(T)+W_{A}(T)+W_{\mathrm{BT}}(T)\right]^{-1} .
$$

Figure 3 includes calculations of the photoluminescence intensity, the photoluminescence lifetime, and photocurrent as a function of temperature, using the parameters listed in Table I. All parameters were derived by fitting the experimental data in Figs. 3 and 4, except for the backtransfer energy, which was taken from Ref. 6 and the Auger quenching coefficient, which was taken from Ref. 5. In the calculations it is assumed that all implanted Er can contribute to the photocurrent.

Figure 3 shows that as the temperature is increased to $100 \mathrm{~K}$ the Er photoluminescence lifetime and intensity decrease slightly with temperature, due to Auger quenching. This effect does not lead to the generation of free carriers, and hence the Er-related photocurrent is negligible in this temperature range. At higher temperatures (above $150 \mathrm{~K}$ ) backtransfer sets in, leading to a further decrease of the luminescence lifetime and intensity. As the temperature increases, the $e-h$ pair dissociation $W_{D}$ starts to be competitive with $e-h$ pair recombination occurring at the Er-related trap level $\left(W_{R}\right)$. The dissociation process generates free carriers in and near the depletion region that can be collected, leading to the buildup of a measurable photocurrent. Finally, when $W_{D}$ becomes larger than $W_{R}$, the Er-related photocurrent is expected to saturate. This effect can be seen in Fig. 3 as a change in the slope of the calculated current data at high temperatures.

In fitting the data, it was found that the values for the parameters $\sigma_{T}, W_{R}$, and $W_{E}$ are correlated. In order to re- 
TABLE II. Comparison of the various rates in the model of Fig. 1, calculated from the steady state solution of the rate equation model, using the input parameters listed in Table I. Results are shown for 15 and $300 \mathrm{~K}$.

\begin{tabular}{lccc}
\hline \hline Rates $\left(\mathrm{s}^{-1}\right)$ & Symbol & $15 \mathrm{~K}$ & $300 \mathrm{~K}$ \\
\hline Radiative decay rate & $W_{0}$ & $1.0 \times 10^{3}$ & $1.0 \times 10^{3}$ \\
Free carrier Auger quenching rate & $W_{A}$ & $\sim 0$ & $1.7 \times 10^{3}$ \\
Backtransfer rate & $W_{\mathrm{ST}}$ & $\sim 0$ & $1.7 \times 10^{6}$ \\
Trap dissociation rate & $W_{D}$ & $\sim 0$ & $5.2 \times 10^{8}$ \\
Trap recombination rate & $W_{R}$ & $2 \times 10^{8}$ & $2 \times 10^{8}$ \\
\hline \hline
\end{tabular}

produce PL lifetime quenching above $150 \mathrm{~K}$, the backtransfer rate has to become significant compared to the Er radiative lifetime, and consequently the prefactor $W_{E}$ has to be larger than $\sim 10^{7} \mathrm{~s}^{-1}$. Furthermore, in order to observe PL lifetime quenching the backtransfer has to be followed by either $e-h$ pair dissociation (scaling with $\sigma_{T}$ ) or recombination at the Er-related trap $\left(W_{R}\right)$. The observation that the photocurrent has not yet saturated at room temperature implies that the $e-h$ pair dissociation is limiting the current generation, and backtransfer followed by recombination at the Er-related trap $\left(W_{R}\right)$ is causing the initial PL lifetime quenching. Within these constraints for $\sigma_{T}, W_{R}$, and $W_{E}$, similar fits can be obtained with either a low $W_{E}$ and a high $W_{R}$ or a high $W_{E}$ and a low $W_{R}$.

The absolute slope of the photocurrent data near room temperature in Fig. 3 corresponds to an activation energy that is lower than backtransfer energy and the dissociation energy. In order to reproduce this behavior, the current generation process must approach saturation near room temperature. This means that at room temperature $e-h$ pair dissociation $\left(W_{D}\right)$ is competitive with recombination at the Errelated trap, which determines the ratio $\sigma_{T} / W_{R}$. Electron capture cross sections in $\mathrm{Si}$ are typically in the range $10^{-14}-10^{-16} \mathrm{~cm}^{2}$. For the fits in Fig. 3 we have chosen $\sigma_{T}$ $=3 \times 10^{-15} \mathrm{~cm}^{2}$.

In order to obtain the correct absolute value of the roomtemperature photocurrent, the product $L_{\mathrm{eff}} \times \eta_{\text {coll }}$ was found to be 9 . Assuming $\eta_{\text {coll }} \approx 1$, this implies that the number of internal reflections is 9, which is a reasonable value for a PERL cell. For a good description of the Auger quenching up to $100 \mathrm{~K}$, a dopant concentration of $3.5 \times 10^{15} \mathrm{~cm}^{-3}$ was found, corresponding to the background doping of the cell. The ionization energy was found to be $30 \mathrm{meV}$, which is smaller than that of boron in $\mathrm{Si}(45 \mathrm{meV})$, which may be attributed to the donor behavior of Er itself, ${ }^{14,17}$ or to the presence of a potential gradient near the edge of the depletion region.

Table II lists various energy transfer rates calculated at 15 and $300 \mathrm{~K}$. As can be seen the backtransfer rate constant increases from a negligible value at $15 \mathrm{~K}$ to $1.7 \times 10^{6} \mathrm{~s}^{-1}$ at $300 \mathrm{~K}$. The dissociation rate constant increases from a negligible value at $15 \mathrm{~K}$ to $5.2 \times 10^{8} \mathrm{~s}^{-1}$ at $300 \mathrm{~K}$. At $300 \mathrm{~K}$ the dissociation rate constant is more than two orders of magnitude larger than the backtransfer rate constant, and more than a factor 2 larger than the recombination rate constant $\left(W_{R}\right)$, showing that the photocurrent is approaching saturation. Note that the backtransfer rate constant at $300 \mathrm{~K}$ is much larger than the radiative rate constant $\left(1 \times 10^{3} \mathrm{~s}^{-1}\right)$ and the free-carrier Auger quenching rate constant $\left(6.5 \times 10^{3} \mathrm{~s}^{-1}\right)$. From the data it can be calculated that at $300 \mathrm{~K}$, the excited Er ions generate free carriers that can be collected with an efficiency of $70 \%$.

\section{IMPLICATIONS FOR SOLAR CELL AND INFRARED DETECTOR DESIGN}

As demonstrated previously, the internal quantum efficiency due to backtransfer at $300 \mathrm{~K}$ is calculated to be $70 \%$, i.e., once a $1.54 \mu \mathrm{m}$ photon is absorbed by an $\mathrm{Er}^{3+}$ ion, the probability that it leads to the generation of an $e-h$ pair that can be collected is $70 \%$. The external quantum efficiency, defined as the number of $e-h$ pairs collected per incoming photon, derived from the photocurrent data amounts to 1.8 $\times 10^{-6}$ at $300 \mathrm{~K}$. This number is low due to the small absorption cross section of the $\mathrm{Er}^{3+}$ ions, combined with the relatively low Er concentration in the sample. In order to have a significant effect on the infrared response of a Si solar cell, the Er concentration in the cell must be significantly increased, or the interaction length of the light with the Er should be strongly enhanced.

In the latter respect it is interesting to note the possibility to use Er-doped $\mathrm{Si}$ in a waveguide detector geometry, in which the light path can be of centimeter length, rather than the micrometer range in the PERL cell discussed in this paper. Experiments on the photoresponse of such Er-doped Si infrared waveguide detectors are under way at this moment. The maximum time response for such a detector at room temperature is given by the slowest of the backtransfer and trap dissociation rates. From Table II it follows that this is equal to $1.7 \times 10^{6} \mathrm{~s}^{-1}$ at room temperature. Detectors with a megahertz bandwidth may be useful in Si-based optoelectronic interconnects in, e.g., high power Si transistor devices in which frequency often is not a critical parameter.

\section{CONCLUSIONS}

PL intensity, PL lifetime, and infrared $(\lambda \approx 1.5 \mu \mathrm{m})$ photocurrent measurements have been performed on an Erimplanted $p-n$ junction in the temperature range $15-300 \mathrm{~K}$. All data can be fitted using a model for the energy transfer processes in Er-doped silicon that is centered around an Errelated trap that mediates the energy transfer between the $\mathrm{Si}$ electronic system and the Er $4 f$ energy levels. The temperature-dependent excitation transfer rates and energies are determined, and it is found that nonradiative recombination at the trap must be included in the model. A significant photocurrent is measured from the junction around 1.535 $\mu \mathrm{m}$, which is attributed to energy transfer from optically excited Er to the Si electronic system. The efficiency of this process at room temperature is $70 \%$. Such energy conversion processes may be useful in Si solar cells and infrared detector technology once the Er concentration or light path length can be significantly increased.

\section{ACKNOWLEDGMENTS}

This paper is a spin-off from a long-standing collaboration between FOM-AMOLF and CNR-IMETEM (Catania). We are very grateful to the late Professor Ugo Campisano, 
who took important initiatives to promote Si-based optoelectronic research in Europe, and who was always a stimulating collaborator in our joint projects. This work is part of the research program of the "Stichting voor Fundamenteel Onderzoek der Materie (FOM),', which is financially supported by the "Nederlandse organisatie voor Wetenschappelijke Onderzoek (NWO).', It is part of the NWOPRIORITY program "Solar Cells for the 21st Century. The authors wish to thank M. J. A. de Dood and J. van der Elsken for valuable discussions, and M. Keevers, A. Wang, J. Zhao, and M. A. Green (University of New South Wales, Australia) and R. Elliman (ANU, Canberra, Australia) for the fabrication of the PERL cells.

${ }^{1}$ J. Michel, J. L. Benton, R. F. Ferrante, D. C. Jacobson, D. J. Eaglesham, E. A. Fitzgerald, Y.-H. Xie, J. M. Poate, and L. C. Kimerling, J. Appl. Phys. 70, 2672 (1991).

${ }^{2}$ S. Coffa, G. Franzò, F. Priolo, A. Polman, and R. Serna, Phys. Rev. B 49, 16313 (1994).

${ }^{3}$ F. Priolo, G. Franzò, S. Coffa, A. Polman, S. Libertino, R. Barklie, and D. Carey, J. Appl. Phys. 78, 3874 (1995).

${ }^{4}$ J. Palm, F. Gan, B. Zheng, J. Michel, and L. C. Kimerling, Phys. Rev. B 54, 17603 (1996).

${ }^{5}$ F. Priolo, G. Franzò, S. Coffa, and A. Carnera, Phys. Rev. B 57, 4443 (1998).
${ }^{6}$ P. G. Kik, M. J. A. de Dood, K. Kikoin, and A. Polman, Appl. Phys. Lett. 70, 1721 (1997).

${ }^{7}$ I. Tsimperidis, T. Gregorkiewicz, H. H. P. Th. Bekman, and C. J. G. M. Langerak, Phys. Rev. Lett. 81, 4748 (1998).

${ }^{8}$ G. Franzò, F. Priolo, S. Coffa, A. Polman, and A. Carnera, Appl. Phys. Lett. 64, 2235 (1994); B. Zheng, J. Michel, F. Y. G. Ren, L. C. Kimerling, D. C. Jacobson, and J. M. Poate, ibid. 64, 2842 (1994); J. Stimmer, A. Reittinger, J. F. Nützel, G. Abstreiter, H. Holzbrecher, and Ch. Buchal, ibid. 68, 3290 (1996).

${ }^{9}$ Silicon Solar Cells, edited by M. A. Green (University of New South Wales, Sydney, 1995).

${ }^{10}$ M. J. Keevers, F. W. Saris, G. C. Zhang, J. Zhao, M. A. Green, and R. Elliman, Proceedings of the 13th European Photovoltaic Solar Energy Conference, Nice, October 1995 (unpublished), p. 1041.

${ }^{11}$ J. Michel, B. Zheng, J. Palm, E. Ouellette, and L. C. Kimerling, Mater. Res. Soc. Symp. Proc. 422, 317 (1996).

${ }^{12}$ D. E. McCumber, Phys. Rev. 134, A299 (1964).

${ }^{13}$ D. E. McCumber, Phys. Rev. 136, A954 (1964); W. J. Miniscalco, J. Lightwave Technol. 9, 234 (1991).

${ }^{14}$ J. L. Benton, J. Michel, L. C. Kimerling, D. C. Jacobson, Y.-H. Xie, D. J. Eaglesham, E. A. Fitzgerald, and J. M. Poate, J. Appl. Phys. 70, 2667 (1991).

${ }^{15}$ A. Taguchi and K. Takahei, J. Appl. Phys. 83, 2800 (1998).

${ }^{16}$ C. D. Thurmond, J. Electrochem. Soc. 122, 1133 (1975).

${ }^{17}$ F. Priolo, S. Coffa, G. Franzò, C. Spinella, A. Carnera, and V. Bellani, J. Appl. Phys. 74, 4936 (1993). 